\title{
PEN as substrate for new solar cell technologies
}

\author{
M. Fonrodona*, J. Escarré, F. Villar, D. Soler, J.M. Asensi, J. Bertomeu, J. Andreu \\ CeRMAE - Departament de Física Aplicada i Òptica, Universitat de Barcelona. Martí i Franquès 1, \\ 08028 Barcelona (Catalonia, Spain)
}

\begin{abstract}
The possible use of poly(ethylene naphthalate) as substrate for thin silicon solar cells has been studied in this paper. The transparency of this polymer makes it a candidate to be used in both substrate and superstrate configurations. $\mathrm{ZnO}: \mathrm{Al}$ has been deposited at room temperature on top of PEN. The resulting structure PEN/ZnO:Al presented good optical and electrical properties. PEN has been successfully textured (nanometer and micrometer random roughness) using Hot-Embossing Lithography. Reflector structures have been built depositing $\mathrm{Ag}$ and $\mathrm{ZnO}: \mathrm{Al}$ on top of the stamped polymer. The deposition of these layers did not affect the final roughness of the whole. The reflector structure has been morphologically and optically analysed to verify its suitability to be used in solar cells.
\end{abstract}

Keywords: PEN, Solar cells, Light trapping, Hot-Embossing Lithography, ZnO:Al

\footnotetext{
* Corresponding author. Tel.: +34 93 4021134; Fax: +34 934021138

e-mail address: mfonrodona@ub.edu (M. Fonrodona)
} 


\section{Introduction}

The use of flexible plastic substrates is becoming an issue of great interest in thin film silicon solar cells technology, as they can contribute to cost reduction in the production process being compatible with the use of roll-to-roll deposition systems and with large area deposition [1]. If we compare their properties with those of flexible metallic substrates commonly used in roll-to-roll processes, polymer substrates turn to be cheaper and to make the serial interconnection between modules simpler. Besides, if the chosen polymer is transparent enough, the configuration of the device is not substrate-limited, as it can be used in both substrate (n-i-p) or superstrate (p-i-n) configurations.

The need to reduce time and costs -thus, to reduce the thickness of the intrinsic layer- has highlighted the role of light trapping strategies, like the use of textured front contacts and/or back reflectors, that allow enhanced light absorption in thinner devices [2]. Additionally, a reduction in the active layer of the devices also enhances the open circuit voltage and the fill factor due to enhanced carrier collection. Light trapping can be achieved by using either a textured Transparent Conductive Oxide (TCO) or a textured substrate (glass or plastic). Furthermore, these benefits arisen from the use of textured substrates to increase light confinement are also promising for the new solar cell technology based on organic semiconductors.

Our goal is the use of poly(ethylene naphthalate) (PEN) as substrate in thin film silicon solar cells. This work is a preliminary technological step in such development focusing, on one hand, on the deposition of TCO layers ( $\mathrm{ZnO}: \mathrm{Al})$ on top of PEN. On the other hand, our approach to light trapping using polymer substrates will be to transfer 
controlled roughness to the PEN substrate by Hot-Embossing Lithography (HEL) aiming to a future use in PEN/metal/TCO/n-i-p/TCO structured solar cells. This imprinting technique allows the reproduction of a surface on a plastic substrate heated above its glass transition temperature ( $\left.\mathrm{T}_{\text {glass }}\right)$. Besides, both the capability to produce repeatable features over a large area and the fact that a given master can be used several times make HEL a very interesting technique $[3,4]$.

The choice of the most suitable plastic substrate was a crucial aspect in this work, as it must be "texturable" by HEL and compatible with our state-of-the-art device-quality silicon thin film deposition. PEN is a semi-crystalline, thermoplastic polyester material, with a $\mathrm{T}_{\text {glass }} \sim 125^{\circ} \mathrm{C}$, but a working temperature up to $155^{\circ} \mathrm{C}$ [5]. It has good optical clarity -what makes it a feasible candidate both p-i-n and n-i-p structures-, and is chemically resistant to most diluted acids and organic solvents [6].

In this paper we present results concerning the optimisation of the structural, optical and electrical properties of $\mathrm{ZnO}: \mathrm{Al}$ layers deposited on $\mathrm{PEN}$, comparing the results with those achieved on glass. Additionally, results regarding random roughness transference on PEN using HEL are also presented. Morphological analysis has been carried out to verify the reproducibility of the master in the stamping process and the conservation of the texture with the subsequent depositions. Reflector structures (PEN/Ag/ZnO:Al) on textured polymer have also been studied optically.

\section{Experimental}

The $\mathrm{ZnO}$ :Al samples have been deposited by rf magnetron sputtering at room temperature, i.e. with no intentional heating, on PEN (0.125 mm-thick) and Corning 


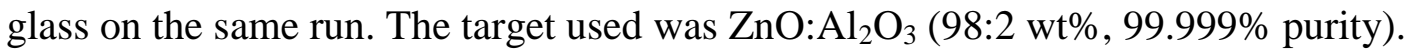

Different $\mathrm{rf}$ power values have been considered using an Ar gas pressure of $3 \cdot 10^{-3}$ mbar. Structure of the samples was analysed by X-Ray Diffraction (XRD) in a BraggBrentano $\theta / 2 \theta$ configuration Siemens D-500 diffractometer. Dark conductivity at room temperature $\left(\sigma_{\mathrm{RT}}\right)$ and Hall measurements were performed to evaluate the electrical properties of the TCO layers.

Imprinting of the polymers was performed using a Jenoptik HEX 01 hot embossing system. In this system, the master, whose surface is to be transferred, is placed on top of the polymer (PEN, 0.125 mm-thick). The entirety is, then, heated above the polymer $\mathrm{T}_{\text {glass, }}$ what causes the polymer to soften, making it able to adopt the master surface after applying a force during a set time. Finally, the polymer-master set is cooled below $\mathrm{T}_{\text {glass }}$ before the master can be carefully released. The observable features transferred on the polymer substrate are, thus, the negative of the texture of the master. Embossing conditions used are listed in Table 1.

Two different masters with random, disordered structures were used, one in the micrometric range (commercially available frosted glass, HF etched to produce the frosted appearance) and one in the nanometric range (Asahi U-type TCO from Asahi Glass Co.). RMS roughness values measured for the masters were $36 \mathrm{~nm}$ for the Asahi-U master, and $2.74 \mu \mathrm{m}$ for the frosted glass one, respectively, as can be seen in Table 3.

Two steps have been considered in the study of the reflector structures. The first approach consisted in a thin Ag layer (220 nm) deposited on the masters and the textured PEN. The second step was to study the whole reflector structure, that is including a $200 \mathrm{~nm}$-thick ZnO:Al layer on top of the PEN/Ag structure. 
Morphological analysis of masters and reflectors was performed by means of Atomic Force Microscopy (AFM, Multimode Extended Nanoscope IIIA, Digital Instruments) and white light interferometry (Wyko NT110, Veeco Metrology), as the roughness of the frosted glass did not allow AFM measurements. The Power Spectral Density (PDS) has been obtained from the Fast Fourier Transform (FFT) of the AFM images, and indicates the weight of the different sinusoidal terms so that their lineal combination corresponds to the analysed surface. The optical analysis was done in a set-up that allows the measurement of the Angular Distribution Function (ADF) of the light scattered by a rough surface [7], using a wavelength of $633 \mathrm{~nm}$. Using this configuration, the ADF is defined as the ratio between the energy measured per solid angle unit $\left(\mathrm{E} / \Omega_{\mathrm{det}}\right)$ and the total energy $\left(\mathrm{E}_{\mathrm{tot}}\right)$. For the random structures analysed, a spherical symmetry around the axis determined by the incident beam is assumed. This symmetry allowed us to determine all the scattered light measuring in a circle around the sample only and, thus, to calculate the reflection haze parameter $\left(\mathrm{H}_{\mathrm{R}}\right)$, which is defined as the ratio between the reflected scattered light and the total reflected light.

\section{Results and discussion}

\subsection{ZnO:Al on PEN at low temperature}

A series of samples has been deposited simultaneously on glass and PEN without any intentional heating of the substrate at $\mathrm{P}=3 \cdot 10^{-3} \mathrm{mbar}$, and changing the $\mathrm{rf}$ power used between 60 and $120 \mathrm{~W}$, what in our case caused a variation in the power density between 3 and $6 \mathrm{~W} / \mathrm{cm}^{2}$. Good adherence to the substrate was observed, regardless the 
substrate considered. Nevertheless, bending of the PEN substrates, not possible in the case of the glass ones, indicated compressive stress. This difference between the two substrates was evidenced as a shift in the (002) peak position in the XRD spectra. Table 2 summarises the information extracted from XRD measurements at 60 and $120 \mathrm{~W}$ : the (002) peak position $(2 \theta)$, the grain size $\left(\mathrm{g}_{\mathrm{s}}\right)$ and the c-lattice parameter (c). Only the peak corresponding to preferential orientation perpendicular to the substrate plane (002) was observable in all cases $\left(2 \theta \sim 34.42^{\circ}\right)$. Estimation of $g_{s}$ resulted in values between 25 and $47 \mathrm{~nm}$, the highest the power used, the largest the grains. This fact could be due to increased surface mobility caused by higher ion bombardment during deposition, what seemed to be more efficient than thermal energy to promote the growth of bigger crystallites. Crystallite sizes of $\sim 45 \mathrm{~nm}$ obtained here are significantly larger than the $28 \mathrm{~nm}$ in [8] for a sample deposited at $90^{\circ} \mathrm{C}$ and the $18 \mathrm{~nm}$ for that at room temperature. The main difference between both experiments is the much higher power density used in our case $\left(6 \mathrm{~W} / \mathrm{cm}^{2}\right.$ vs. $\left.0.22 \mathrm{~W} / \mathrm{cm}^{2}\right)$. Besides, no significant difference in the grain size was observed between the two substrates considered, especially at high power.

The shift in the (002) peak position was mainly attributed to higher stress of the layers deposited on glass, which cannot release their internal stress by significantly deforming the substrate. This shift was more pronounced in those samples deposited at higher rf power, what was consistent with a general trend in physical deposition processes, to increase the stress of the samples by increasing the energy of the particles. Taking a deeper look at the crystalline structure of the samples, the c-lattice parameter value obtained on PEN (5.210 $\AA$ at $60 \mathrm{~W}$ and $5.189 \AA$ at $120 \mathrm{~W}$ ) was much closer to the theoretical one $(5.206 \AA)$ than that measured on glass $(\sim 5.220 \AA)$ what could be related to the higher stress mentioned in this case. 
The structure of the samples seemed to influence their optical properties. The optical transmission spectra of the samples deposited at 60 and $120 \mathrm{~W}$ (the lowest and highest analysed, respectively) are shown in Fig. 1, together with the spectrum of plain PEN plotted for comparison. The sample deposited at $120 \mathrm{~W}$ had a higher transmittance in the range comprised between 400 and $500 \mathrm{~nm}$. As this sample is thicker (360 nm-thick) than that deposited at $60 \mathrm{~W}(230 \mathrm{~nm})$ and showing lower transmittance, we could conclude that the optical absorption of the former was significantly lower than that of the one deposited at $60 \mathrm{~W}$. Additionally, and by looking at the transmittance values measured, we could conclude that, optically, the PEN/ZnO:Al structure can be used as substrate in p-i-n solar cells, i.e. with the incoming light going through the substrate.

Finally, the electrical properties of the layers were also analysed as they have a major influence on the final performance of the solar cell. The conductivity at room temperature increased with increasing rf power on both substrates, from $\sigma_{\mathrm{rt}} \sim 200$ $\Omega^{-1} \cdot \mathrm{cm}^{-1}$ at $60 \mathrm{~W}$ to a highest value of about $1500 \Omega^{-1} \cdot \mathrm{cm}^{-1}$ on glass and $1400 \Omega^{-1} \cdot \mathrm{cm}^{-1}$ on PEN at $120 \mathrm{~W}$, as illustrated in Fig. 2. Hall measurements performed on glass resulted in good mobility $(\mu)$ and carrier density values $(\mathrm{n}): \mu=19 \mathrm{~cm}^{2} \cdot \mathrm{V}^{-1} \cdot \mathrm{s}^{-1}$ and $\mathrm{n}=$ $5 \cdot 10^{20} \mathrm{~cm}^{-3}$ at $120 \mathrm{~W}$ and $\mu=7 \mathrm{~cm}^{2} \cdot \mathrm{V}^{-1} \cdot \mathrm{s}^{-1}$ and $\mathrm{n}=1.8 \cdot 10^{20} \mathrm{~cm}^{-3}$ at $60 \mathrm{~W}$, respectively. From these results, we could conclude that the electrical properties obtained also pointed to the feasibility of the future use of PEN/ZnO:Al structures as substrates for solar cells.

\subsection{Texturing PEN by Hot Embossing Lithography}


A standard approach in light confinement is the use of textured TCO on a flat substrate [9]. An alternative approach is the use of textured substrates, which has been yet tested on glass [10] and on poly(ethylene terephthalate) (PET) using photolithographic processes or plasma etching of the PET substrate [11]. In our case, the goal of our study was to transfer controlled roughness to PEN by Hot Embossing Lithography. The stamping conditions used to reproduce the master texture are shown in Table 1. As it can be seen there, slightly higher embossing conditions (higher applied force) were necessary to reproduce the nanometric scale texture of Asahi-U.

Masters and stamped polymers have been morphologically analysed to check the efficiency of the transfer process. Fig. 3 shows the interferometry and AFM images of the masters and of PEN textured using both masters. A comparison between the stamped PEN surfaces and those of the masters used in the process showed similar aspect. No significant difference was observed in the RMS roughness values, presented in Table 3, between master and stamped polymer, either having used frosted glass (roughness of a few micrometers) or Asahi-U (roughness of tens of nanometers), taking into account that the stamped surface is the negative of the master's. It is observable in Fig. 3 that Asahi-U's surface was not optimally transferred on the PEN. This fact can be attributed to some problem during the release of the master. If smaller selected areas were analysed -as shown in the PSD graphs in Fig. 4-, good transfer and better agreement between RMS roughness values was reached. In Fig. 4a, looking at the PSD corresponding to the images in Fig. 3 (textured PEN and negative of the master, $5 \mu \mathrm{m} \times$ $5 \mu \mathrm{m})$, good agreement was observed for $\lambda \geq 1 \mu \mathrm{m}$, indicating that structures with this periodicity were correctly transferred. Conversely, when a smaller surface was analysed (Fig. $4 \mathrm{~b}, 1 \mu \mathrm{m} \times 1 \mu \mathrm{m}$ ), the curves from master and textured polymer were much 
similar, evidencing a good correspondence between both surfaces. These troubles could be avoided by some anti-adhesion coating of the master.

Results shown indicated the viability to use hot embossing as imprinting technique to reproduce a random texture on PEN, whose working temperature is compatible with silicon deposition, regardless the dimensions of the roughness used. The viability to use HEL to reproduce random roughnesses on polymers more commonly used in imprinting process like poly(methyl methacrylate) (PMMA) has already been demonstrated [7].

\subsection{Reflectors}

The morphological effect of the deposition of the two layers constituting the reflector (Ag and $\mathrm{ZnO}: \mathrm{Al}$ ) was studied independently. In Table 3, the RMS roughness values obtained after the deposition of a $220 \mathrm{~nm}$-thick Ag layer on top of the masters and textured PEN can be seen. The same values measured after the deposition of $200 \mathrm{~nm}$ of $\mathrm{ZnO}: \mathrm{Al}$ on top of the Ag layer are also presented in Table 3. In general, good agreement is found between the values obtained in all cases, RMS roughness values around $40 \mathrm{~nm}$ were measured when the master considered was Asahi-U, and close to $2.8 \mu \mathrm{m}$ when frosted glass was used. The slight disagreement seen in the value measured on PEN textured using frosted glass coated with Ag can be attributed to the fact that the surfaces measured did not correspond to the same point or to the part of the master facing the polymer in the same point. Therefore, it could be concluded that the deposition of an $\mathrm{Ag} / \mathrm{ZnO}: \mathrm{Al}$ reflector structure on top of the stamped PEN did not affect significantly the roughness of the whole, regardless the roughness size considered. 
Fig. 5 shows the ADF of both masters coated with $\mathrm{Ag} / \mathrm{ZnO}: \mathrm{Al}$ and of PEN textured with Asahi-U and frosted glass, respectively, also coated with Ag/ZnO:Al. Table 4 shows the $\mathrm{H}_{\mathrm{R}}$ values for the different structures studied. Analysing the ADF figures, it can be seen that no significant difference was observed in the case of the frosted glass between positive (master) and negative (textured polymer) of a certain texture. In fact, $\mathrm{H}_{\mathrm{R}}$ was almost 1 (all light scattered) in all cases where this master was used, and this value did not depend on the subsequent deposition of $\mathrm{Ag}$ and $\mathrm{ZnO}$ :Al layers on top of the rough surface. On the other hand, a decrease in the ADF values between positive and negative was observed with Asahi-U texture. In this case, we found that much less light was scattered using nanometer-size roughness, presenting values around $\mathrm{H}_{\mathrm{R}}=0.3$ when the structure substrate/Ag was analysed. The reflection haze value in the same structures once $\mathrm{ZnO}: \mathrm{Al}$ had been deposited on top of the Ag layer dramatically increased in both cases (from 0.36 to 0.69 for the master and from 0.32 to 0.51 in the case of the textured PEN). A change in the actual light wavelength to one closer to the dimensions of the roughness of the Asahi-U surface when crossing the $\mathrm{ZnO}: \mathrm{Al}(\mathrm{n} \sim 2)$ could be the cause of such a large increase.

Results up to now suggest that back reflectors deposited on top of PEN textured using HEL can be used as substrates for solar cells. Further work needs to be done to identify the optimal roughness in every situation, depending on the material considered and on the thickness of the active layer of the solar cell.

\section{Conclusions}


The viability to use PEN as substrate for solar cells has been studied in this paper. ZnO:Al layers have been deposited on PEN and glass by rf magnetron sputtering at room temperature. Optical transmittance measurements indicated that the PEN/ZnO:Al structure is suitable to be used in solar cells in the superstrate configuration. Moreover, the electrical values obtained also pointed in the same direction.

Hot-Embossing Lithography has been proven as a viable technique to reproduce random textures (nanometer and micrometer size) on PEN.

Reflector structures heading to a future use as substrates in solar cells where done depositing Ag and $\mathrm{ZnO}$ :Al on top of the stamped polymer. The deposition of these layers did not affect the final roughness of the whole. The reflector structure consisting in textured PEN coated with $\mathrm{Ag} / \mathrm{ZnO}: \mathrm{Al}$ was morphologically and optically suitable to be used in solar cells.

\section{Acknowledgements}

This work has been financed by the Spanish Government (MAT2001-3541-C03-01). The authors also want to thank the Scientific-Technical Services of the Universitat de Barcelona for the AFM, XRD and SEM measurements, and Dr. Chris Mills from the Laboratori de Recerca en Nanobioenginyeria del Parc Científic de Barcelona for the stamping of the polymers and the interferomentry measurements.

\section{References}

[1] M. Izu, T. Ellison, Sol. Energy Mater. Sol. Cells 78 (2003) 613 
[2] J. Morris, R.R. Ayra, J.G. O’Down, S. Wiedemen, J. Appl. Phys. 67 (1989) 1079

[3] R.W. Jaszewski, H. Shift, J. Gobrecht, P. Smith, Microelectron. Eng. 41/42 (1998) 575

[4] J.L. Charest, L.E. Bryant, A.J. Garcia, W.P. King, Biomaterials 25 (2004) 4767

[5] T. Higashioji, T. Tsunekawa, B. Bhushan, Tribol. Int. 36 (2003) 437

[6] I. Ouchi, I. Nakai, M. Kamada, Nucl. Instrum. Meth. Phys. Res. B 199 (2003) 270

[7] J. Escarré, F. Villar, M. Fonrodona, D. Soler, J.M. Asensi, J. Bertomeu, J. Andreu, Sol. Energy Mater. Sol. Cells (2004) accepted for publication

[8] D.H. Zhang, T.L. Yang, J. Ma, Q.P. Wang, R.W. Gao, H.L. Ma, Appl. Surf. Sci. 158 (2000) 43

[9] J. Müller, B. Rech, J. Springer, M. Vanecek, Sol. Energy (2004) in press

[10] N. Chuansuwanich, P.I. Widenborg, P. Campbell, A.G. Aberle, Proc. of $14^{\text {th }}$ PVSEC (2004) 325

[11] V. Terrazzoni-Daudrix, J. Guillet, X. Niquille, L. Feitknecht, F. Freitas, P.

Winkler, A. Shah, R. Morf, O. Parriaux, D. Fisher, Proc. of $3^{\text {rd }}$ WCPEC (2003) 1596 


\section{Figure captions}

Fig. 1. Optical transmission spectra of the $\mathrm{ZnO}$ :Al layers deposited at 60 and $120 \mathrm{~W}$ on PEN. Plain PEN spectrum is included for comparison.

Fig. 2. Dark conductivity at room temperature as a function of the RF power on glass and PEN.

Fig. 3. White light interferometry images of frosted glass master (a) and of frosted glass textured PEN (c), and AFM images of Asahi-U master (b) and of Asahi-U textured PEN (d). The circle in (d) corresponds to the small area studied in Fig. 3.

Fig. 4. PSD of different sizes of the negative of the Asahi-U master surface and of textured PEN.

Fig. 5. ADF of Ag/ZnO:Al coated Asahi-U master and textured PEN (top) and frosted glass master and textured PEN (bottom). 


\section{Table captions}

Table 1. PEN stamping conditions for the two masters considered.

Table 2. Information extracted from XRD measurements of $\mathrm{ZnO}$ : $\mathrm{Al}$ layers deposited on glass and on PEN.

Table 3. Summary of the roughness RMS values for all structures considered.

Table 4. Reflection haze parameter values for all the reflector structures considered. 
Fig. 1

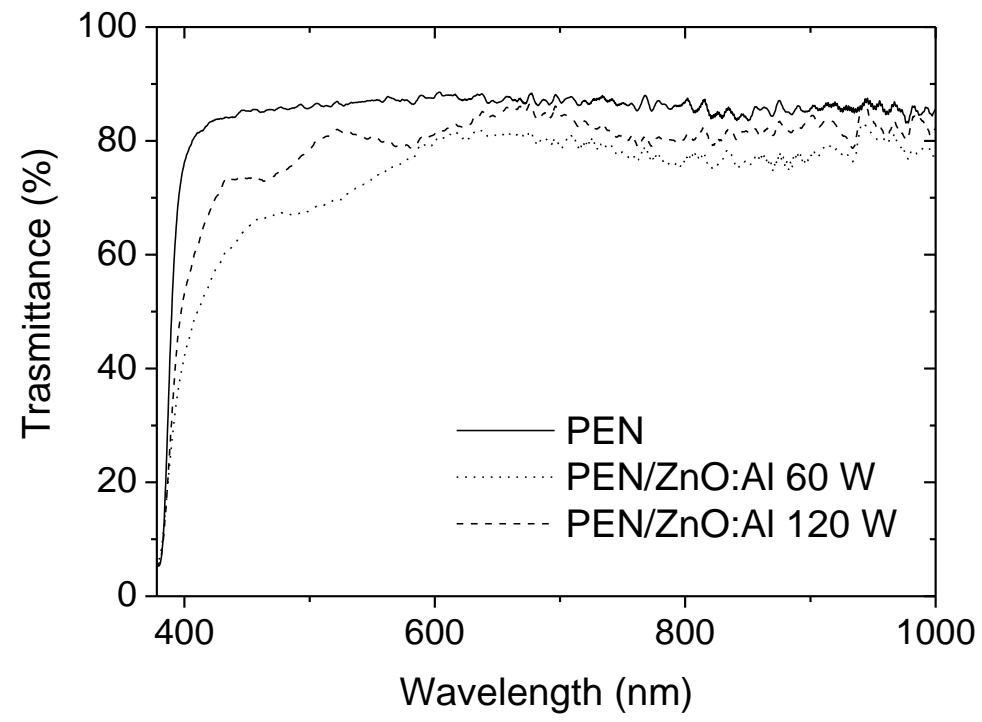


Fig. 2

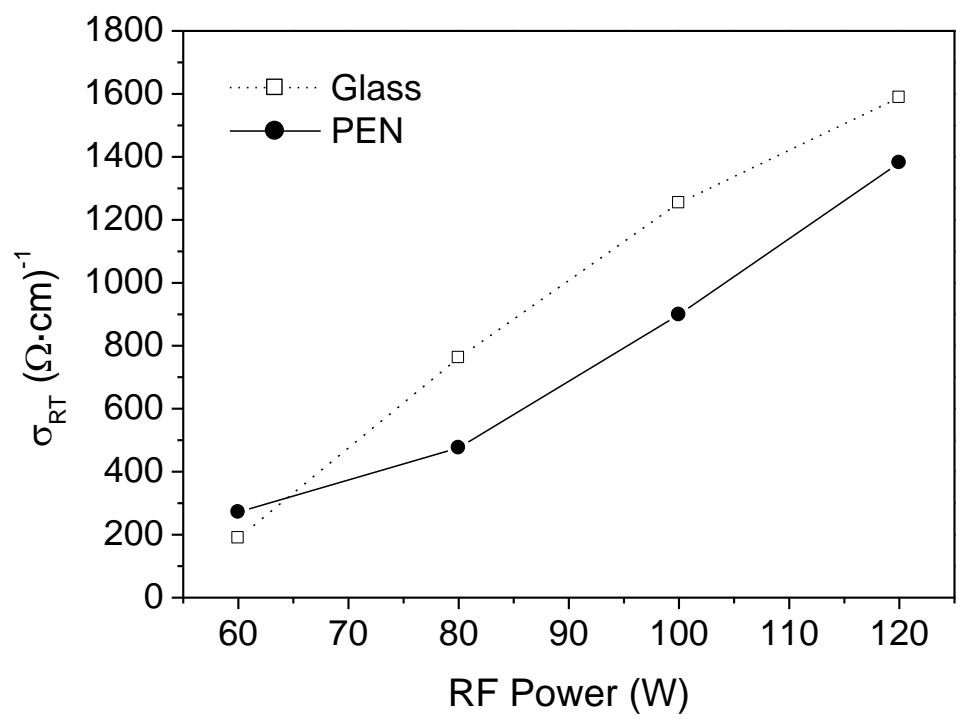


Fig. 3

a)

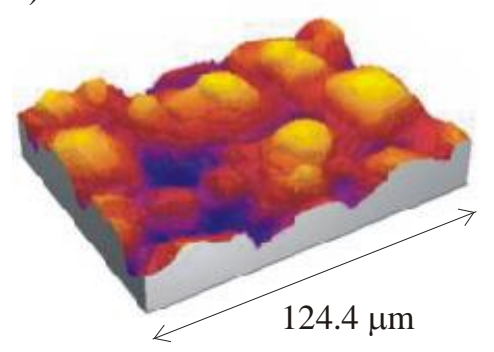

c)

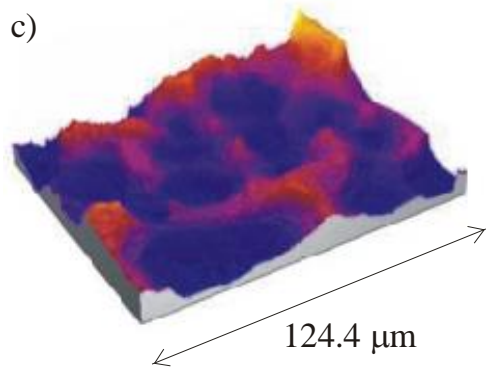

b)

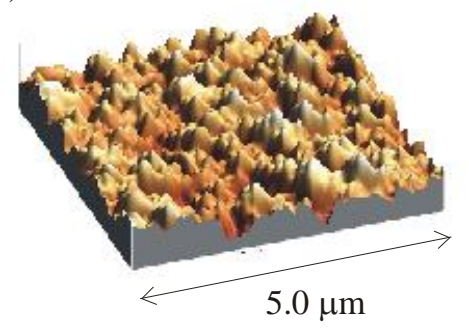

d)

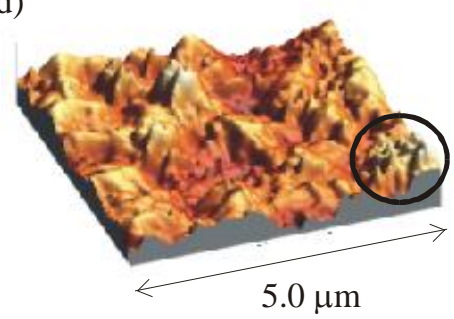


Fig. 4
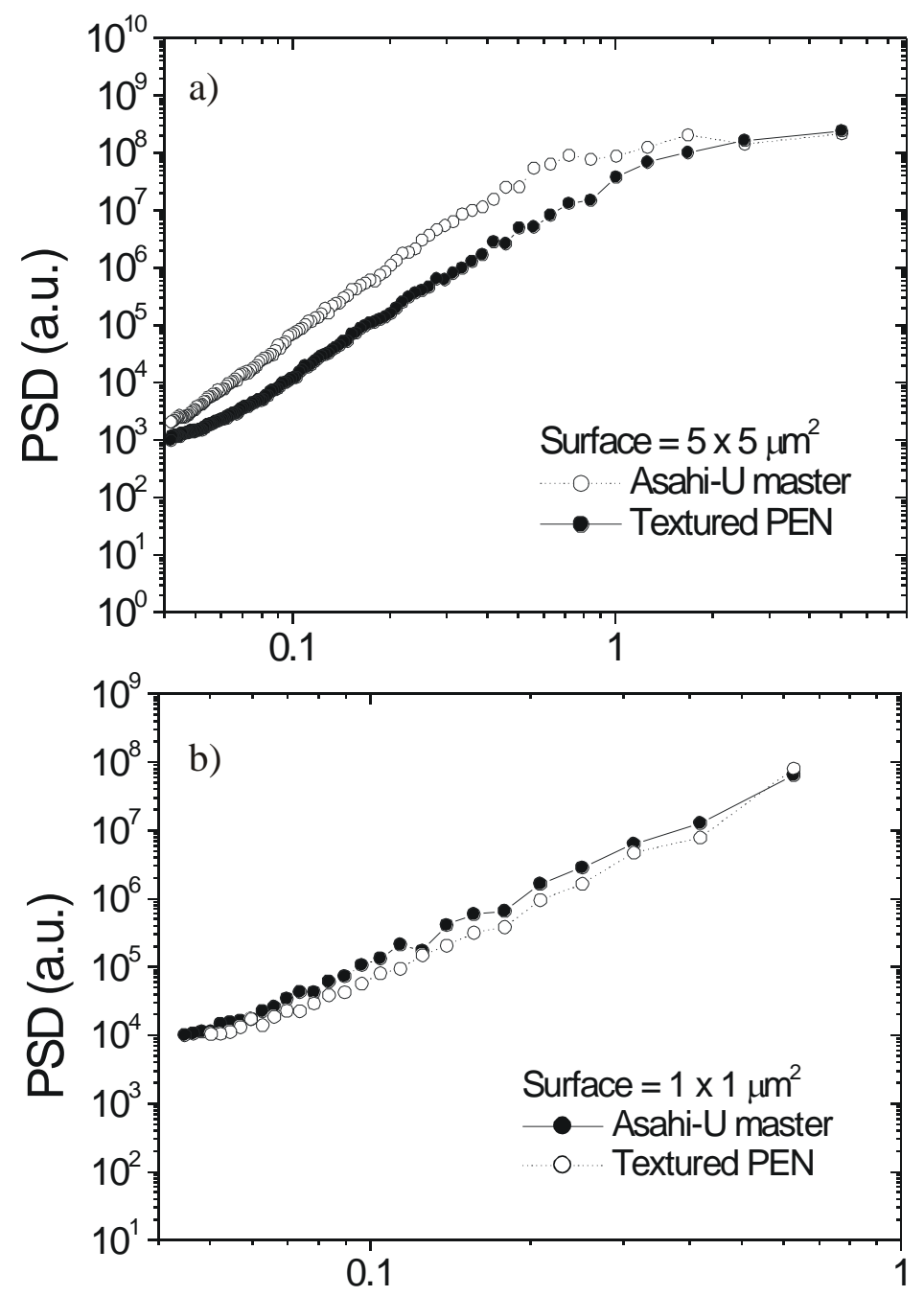

Wavelength $(\mu \mathrm{m})$ 
Fig. 5

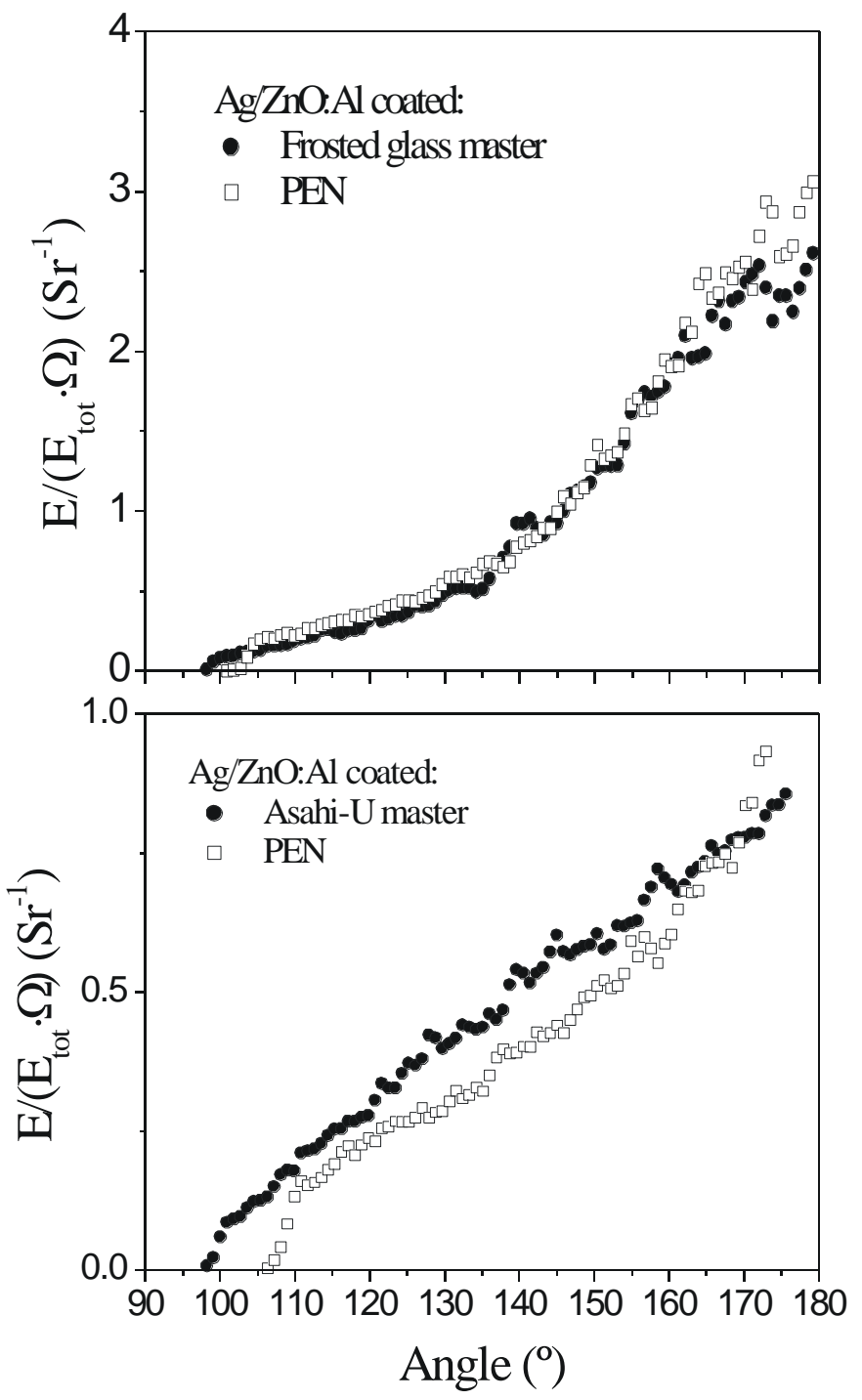


Table 1

\begin{tabular}{cccc}
\hline Master & $\mathbf{T ~}\left({ }^{\mathbf{0}} \mathbf{C}\right)$ & $\mathbf{P}\left(\mathbf{N} / \mathbf{m}^{2}\right)$ & $\mathbf{t}(\mathbf{s})$ \\
\hline Frosted glass & 200 & $2.0 \cdot 10^{7}$ & 1200 \\
\hline Asahi-U & 200 & $3.0 \cdot 10^{7}$ & 1200 \\
\hline
\end{tabular}


Table 2

\begin{tabular}{ccccc}
\hline Substrate & RF Power $(\mathbf{W})$ & $\mathbf{2 \theta}\left(^{\mathbf{0}}\right)$ & $\mathbf{g}_{\mathbf{S}}(\mathbf{n m})$ & $\mathbf{c}(\AA)$ \\
PEN & 60 & 34.43 & 36 & 5.210 \\
& 120 & 34.57 & 47 & 5.189 \\
\hline \multirow{2}{*}{ Glass } & 60 & 34.35 & 25 & 5.221 \\
& 120 & 34.36 & 44 & 5.220 \\
\hline
\end{tabular}


Table 3

\begin{tabular}{cccc}
\cline { 3 - 4 } & & \multicolumn{2}{c}{ RMS roughness (nm) } \\
& plain & Frosted glass & Asahi-U \\
& & 2740 & 36 \\
master & + Ag & 2680 & 45 \\
& + Ag/ZnO:Al & 2950 & 43 \\
\hline \multirow{2}{*}{ PEN } & textured & 2980 & 26 \\
& + Ag & 2080 & 37 \\
& + Ag/ZnO:Al & 2850 & - \\
\hline
\end{tabular}


Table 4

\begin{tabular}{cccc}
\cline { 3 - 4 } & & \multicolumn{2}{c}{$\mathbf{H}_{\mathbf{R}}$} \\
& & Frosted glass & Asahi-U \\
\hline \multirow{2}{*}{ master } & $+\mathrm{Ag}$ & 0.999 & 0.36 \\
& $+\mathrm{Ag} / \mathrm{ZnO}: \mathrm{Al}$ & 0.999 & 0.69 \\
\hline \multirow{2}{*}{$\mathrm{PEN} \quad+\mathrm{Ag}$} & 0.999 & 0.32 \\
& $+\mathrm{Ag} / \mathrm{ZnO}: \mathrm{Al}$ & 0.999 & 0.51 \\
\hline
\end{tabular}

